\title{
Perspectives on Online and On-Site Pedagogy: The Impact of Technology Now and in the Future
}

\author{
Marion G. Ben-Jacob ${ }^{1}$, Tyler E. Ben-Jacob ${ }^{2}$ \\ ${ }^{1}$ Department of Mathematics and Computer Information Science, Mercy College, New York, USA \\ ${ }^{2}$ Medical School, American University of Antigua, University Park, USA \\ Email: mbenjacob@mercy.edu, relyt616@yahoo.com
}

Received June $3^{\text {rd }}, 2013$; revised July $5^{\text {th }}, 2013$; accepted July $29^{\text {th }}, 2013$

\begin{abstract}
Copyright $\odot 2013$ Marion G. Ben-Jacob, Tyler E. Ben-Jacob. This is an open access article distributed under the Creative Commons Attribution License, which permits unrestricted use, distribution, and reproduction in any medium, provided the original work is properly cited.
\end{abstract}

\begin{abstract}
Technological advances of this millennium have allowed for enhanced learning experiences for students. Technology has and continues to be integrated into the educational environment from many perspectives and to different degrees with the use of different electronic tools. This paper discusses the capabilities and uses of the more popular tools and some of the pedagogical issues that both faculty and students need to be cognizant of when using these educational boons. The effects on life-long learning and global responsibility are discussed. The perspectives of on-site and online learning are addressed with regard to teaching strategies and goals. An understanding of the impact technology will have in the future is discussed as well.
\end{abstract}

Keywords: Pedagogy; Technology; Life-Long Learning; Multi-Valued Logic

\section{Introduction}

Advance in recent technologies in communication and computing affect pedagogy and learning. The influence of technology on the learning environment of this millennium cannot be over emphasized. Courses of study that are completely online, hybrid, blended, and even those onsite rely to some degree on technology, be it via research assignments or online tutorials. With the integration of technology into pedagogy come many benefits but concerns as well, as with any significant measure of progress. This paper will present many of the latest advances with regard to pedagogy as a result of technology as well as some of the issues. It will present a multi-valued logic as a necessity for understanding the impact of future technology on learning.

One of the major overarching benefits of technology is that it affords the world the opportunity to engage in global learning. Global learning allows for learning 24/7. It is multicultural and intercultural. It supports tolerance and diversity. It engages those involved with varied perspectives on politics, religion, ethnicity, and race. In addition to increasing the knowledge of the students involved in the specific subject matter, it is a stepping stone for universal acceptance and peace.

Learning is social. Learning is based on different opinions and discussion. Learning is forming inferences as a result of giving rational thought to different sources of information and arriving at conclusions. The integration of technology into pedagogy is sometimes referred to as virtual learning. This can include using Google to search for an answer, reading an e-text, or taking a course online.

\section{Technology}

Onlinelearning platforms, e.g. Blackboard, allow for asyn- chronous learning supported via the posting of lecture notes, a discussion area, and a synchronous chat room. One is able to post announcements, interact via email and make use of tools for the facilitation of grading and tracking of student posts ("Blackboard", 2013). Some of the pedagogical benefits afforded by online learning include time for students to think before responding, usually greater participation as students tend to be less shy online, and easy means of assessment as the course progresses. Online learning is no longer a glorified correspondence course. The lecture notes need not be of the standard variety because technological advances have led the online instructors to incorporate the use of such tools as Adobe Flash Player, software for integrating animation, interactivity, and video into the coursework (Adobe, 2009). Software such as Adobe Connect or Elluminate allow for note writing inadvance, text messaging, interactive whiteboard-import images and PowerPoint slides, illustration in real time with built-in drawing tools, clear, two-way audio over the Internet, and polling features for interactivity as well (Adobe, 2013; Elluminate, 2013).

Blogs, short for web logs, are another technological tool being integrated into pedagogy. They are onlinemessages ordered by the time of posting. They support text, links, images and media. An initial message is posted and readers can add their comments to the posts. They provide a mechanism for reflection and support learning through writing, peer-to-peer sharing of knowledge, knowledge communities and provide a mechanism for faculty to use for communication of coursework (Blogs, 2009; Levin, 2008).

Clickers are another popular technology tool that has been integrated into the pedagogical environment. Students can select their answers to multiple choice questions. These answers can be identified with each student individually or just collected 
as group responses. Clickers can be used for surveys, multiple choice tests, and recently, for formative assessment of student learning (iclicker, 2008).

Wikis are collaborative websites where the content can be edited by anyone who has access to the site. Many professors use wikis for communicating with their on-site students as opposed to developing a blended course or making use of a tool such as Blackboard. Wikis support browser based viewing and editing and tracking of changes. Wikis support group work and peer review and editing. They allow for assembly of notes and shared information. They support faculty collaboration for research, projects, and course development. Students can use wikis for the creation and editing of lecture notes collaboratively, for community building, and for group projects. It is interesting to note that in general students are reluctant to change each other's texts and instead create their own pages. Some of the more well-known wiki sites include PB wiki, and Wetpaint, both of which have no fee associated with them. The most famous wiki is Wikipedia (Levin, 2008; Wikipedia, 2011).

Twitters can be considered a combination of blogging, social networking, and text messaging. The emphasis is on brevity. One is supposed to tweet one's answer, i.e. respond in 140 characters or less. People tweet via text messages on cell phones and on twitter sites, such as Twitter (Twitter, 2009).

Students also have the ability to "follow" people on Twitter, and as such, receive messages that allow them to be aware of the status updates and posts of their peers. E-Portfolios are an electronic means of collecting one's work over a period of time. One can design one's own portfolio with folders and subfolders as necessary. Most e-portfolios systems have a set of rubrics associated with the courses for which a student is using the portfolio. This facilitates the assessment of a student's work and provides a mechanism for the professor to supply formative assessment to his students (Taskstream, 2011).

Given all these advances in technology that facilitate teaching and learning come the associated issues that educators must address and raise the awareness of students as well. These include the fact that user-created content is subject to mistakes, bias, and inconsistencies. Anyone can publish on the Internet and so one must be careful of the validity of the source. Internet resources are temporal. Plagiarism is more rampant and electronic sources must be documented. The form for documentation is different from those of hard copy sources, journals, and books. Blog comments can be volatile and wikis can be modified inappropriately. We need to remember that there are still some students not comfortable with technology as well.

In order to compete in today's educational market, colleges and universities are integrating more and more technology into their environments. The students of this generation have grown up with the technology and most are quite comfortable with it. Pedagogues need to enhance their teaching with technology without losing site of the basic long term goals of educating our students to become global citizens of the world.

Technology tools can be and are being incorporated into both on-site and online classes. In addition to this, the other similarities between on-site and online classes are content material and often textbooks. The goals of both venues are identical as well. We want to prepare our students for global responsibility and life-long learning.

\section{Life-Long Learning}

Life-long learning is the ongoing development and en- hancement of knowledge and skills needed for continuing and changing professional goals and personal satisfaction. The United States Department of Labor estimates that today's student will have $10-14$ jobs by the time s/he reaches the age of 38. The predication is that 1 out of 4 workers today is working for a company s/he has been employed by for less than one year. More that 1 out 2 people are working at their current job for less than 5 years. The top 10 most popular jobs in 2010 did not exist in 2004. These statistics beckon the question for educators - How do we prepare our students for 14 different jobs, several different careers? How do we educate our students for jobs that do not yet exist using technologies that have not yet been invented to possibly solve problems of which we are not even aware (Davis, 2010)?

As previously mentioned, global learning is an educational phenomenon that goes beyond national boundaries. It is concerned with intercultural and international aspects of education as well as specific content areas. Technological advances of this millennium have allowed for students from different countries, backgrounds, and cultures to study and learn together and enhance their educational experiences. Global learning is critical for students in order to embrace world cultures and events and develop as citizens of the world. Global learning can be considered the attainment of knowledge that surpasses national boundaries. Connections between subject matter and future careers and society are formed. It is progress toward the unity of man as well as enhancement of content learning. The goals of global learning include the generating of new knowledge about global studies, the spurring of greater civic engagement and social responsibility, and the cultivation of intercultural skills. Global studies incorporate the mastery of a specific discipline or field (Ben-Jacob, 2011).

Many innovations in today's educational environment incorporate technology to some degree. Indeed, technology has created a global learning community. Technology has facilitated greater collaboration and dialogue among participants who are interested in advancing global learning and learning in general.

Other than the aforementioned similarities, our contention is that analogies between the two venues should not be made. Those who wonder how to compensate in a distance learning class for lack of face-to-face contact which is evident are "missing the mark" with regard to distance learning. Distance learning is a viable environment for student learning and the best pedagogical strategies need to be developed independent of those strategies for teaching in the traditional classroom.

The accelerated growth in technology, demographics, geography, financial considerations of institutions of higher education, but most of all the modus operandi of the student of today have catapulted distance education into the educational environment. The typical college student of today is an individual with a substantial number of commitments in addition to pursuing an education. This student is mature, financially selfsupporting with family responsibilities and really wants to further his education. This prototype of students can not reside on campus and does no event have the time to commute to campus. Without distance learning vast numbers of students will not be able to further their education.

Maturity and motivation are essential qualities for success in this model of learning. An integral component of higher education today is collaborative work, not only because it supports active learning, but also, because it is required for the work- 
place for which we will prepare our students. The fusion between learning and work will be closer than ever before. The ability to express oneself is so critical as the interaction will be necessary between learners and learners, as well as between learners and instructors.

Online learning supports the learners' active questioning of instructors and content experts, as well as the receiving of information from them. It encourages the learners' active exploration and manipulation of material. This aspect of the model is not entirely new. It aligns well with problem-based learning. Problem-based learning proceeds according to the following algorithm: first, the students are presented with the problem. Next, they attempt to solve it using the reasoning skills they possess. Interactively, they identify their learning needs. Then, they study and apply the new knowledge to the problem and finally, they describe what they have learned. In this environment the professor becomes an advisor rather than a lecturer. As an example, in many medical schools students are presented with a clinical case they are to discuss amongst their peers with a professor present to advise. There are no right or wrong answers, but rather the process of investigation and the action of discussing and finding points seemingly relevant are critical to the development the students' abilities. This educational milieu of distance learning supports a collaborative process among its participants with inquiring minds.

A further aspect of today's learner is that he will be a lifelong learner. As the 20th century drew to a close, nearly half of all students enrolled in post-secondary education were adults. If we add to this the numbers of individuals accessing post-secondary level training sponsored or funded by their employers, well over half of today's post-secondary students are adults. Trends suggest that this will increase. Multiple careers and the dependence of more jobs on rapidly changing technology mean more adults will seek additional educational opportunities. Attitudes will continue to change with most people placing a greater value on continued education and perceiving themselves as lifelong learners. With the aid of technology mediated learning, especially distance learning, not only will more adult learners have access to an ever widening array of post-secondary programs, but institutions will transform themselves to serve the pedagogical as well as the lifestyle needs of the lifelong learner.

\section{Pedagogy and Learning}

There are three tenets of the traditional model of post-secondary education that have led to pedagogical strategies that are not particularly suited to the adult learner. Learning is primarily an individual matter. Learning, at least academic learning, is achieved, in large part, through the transfer of knowledge from experts to novices. Learning that can be garnered from individuals' experiences provides little benefit toward academic learning, in spite of the fact that academic learning can inform and enrich individuals' life experiences.

The view that learning is individual has lead professors and institutions to devalue collaboration. Assessing individual contribution to collaborative work raises difficulties for many. This line of thinking, however, fails to ask whether more learning and better learning occurs when learners work individually or collaboratively. Working collaboratively with one's peers exposes each learner to a wider array of questions and perspectives than working as a single learner. Students can learn from their peers and learning is reinforced as they share learning and even teach their peers. Collaborative learning is especially important for the adult learner and for the learner of the 21 st century. As a full time working person, most adult learners are already comfortable working in teams to "get the job done." At work they are encouraged to question team members, share ideas, and produce collectively. Their job performance is judged in part by the performance level of the team. Academic programming designed for adults should take note of how adults work best, not how academics themselves have learned. The workplace of the 21 st century will be increasingly complex and technologically dependent. Corporations will no longer value simply the knowledge and ability to learn of its individual employees (individual learning). Individuals and teams will be valued for their ability to communicate this knowledge and learning in ways that help other employees and teams use this knowledge and enhance the ability of the entire organization to operate smarter and more efficiently (organizational learning).

The view of learning as information and knowledge transfer encourages an emphasis on lectures and reading as the major learning activities within the academy. For the student these are passive activities. Learning, however, occurs with the active manipulation and exploration of content materials. Learning is a process of discovering knowledge, not merely relaying facts. Ideally, this process of discovery is a collaborative one.

The online professor needs to supply notes and ask provocative questions to the students. The students need to collaborate and draw information from the material and each other. Technological tools are compatible with most distance learning platforms. The students in an online class need to be able to formulate questions. They need to be able to be adept at critical inquiry, reading with comprehension, and writing grammatically as a beginning. Using present day technology, the achievement of an education can be done more on one's own time than previously. A student who masters the material quickly can move ahead and the student who needs more repetition can do so without the embarrassment of holding the class back. The actions are in the hands of the students; they need to have the drive and commitment to complete the course.

As pedagogues, we want to insure that our students are provided with the best opportunities for learning. Different strategies and approaches prevail today but given the integration of technology into our lives and our global learning environment it becomes imperative that learning be it in the traditional classroom or online support life-long, learning and global responsibility.

Having acknowledged the aforementioned changes for today's education environment, deliberation must be given to the future as well. Agreement is widespread that technology is here to stay but how it will be enhanced and the effect on education in the future necessitates discussion.

Critical thinking and logical inference will be a stronger than ever influence in future education. Among the startling statistics that support these are: 1) It is estimated that 1.5 exabytes $\left(1.5 \times 10^{18}\right)$ of unique new information will be generated worldwide this year, more than in the previous 5000 years, 2) There are approximately 540,000 words in the English language; about five times as many as during Shakespeare's times (Davis, 2010). With the influx of all the information that will be broadcast via the Internet, decision making processes will be critical. In addition, computation and analysis will be done in parallel. 


\section{Multi-Valued Logic}

Undergraduate students usually study propositional and predicate logic. Most logic systems are based on a two-valued truth system, true and false. Our contention is that today's world is replete with multitasking and parallelism. Parallelism, especially in technology, encourages us to examine the meaning of different logical operations being done concurrently. A question of reasonable complexity is how one would attempt to solve more than one quantitative problem at the same time or attempt to make more than one logical inference concurrently. This question extends itself to non-technological environments as well. This is exemplified by a situation where a problem is subdivided into components with different people working on different components or some working on the same component. We need to give meaning to truth systems that possess the capability of concurrency. Students who use technology, most all students, need to understand how a computer can arrive at a conclusion. Given that sound results can be obtained, we need to specifically understand how logical inferences are made by machines or operations in general that support parallelism. A fundamental goal becomes the understanding of parallel logic.

Existing results credit a greater complexity to parallel logic thinking than information just being distributed over a number of sites. Parallelism, accounting for hardware and supporting language, allows for shared memory, sometimes somewhat restricted, sometime global, and interaction among the processors prior to the final assignment of truth values. We need a five-valued logic. The first four truth-values are: true (t), false (f), undefined $(\stackrel{\perp}{)}$, and suspend (s). The fifth value, overdefined $(\mathrm{T})$, is included for topological facility (Belnap, 1977).

The necessity of the first two truth values in our logic is obvious. The truth value suspend reflects the fact that work has been attempted to establish the truth value of the instantiated predicate in question, no (exact) precise truth valued has yet been assigned and at this point in time work must be stopped and recorded (suspended) so as not to interfere with the validity of the calculations of the other processors. Also, should there be a malfunction in the hardware allowing for one instantiated predicate to be assigned true by one processor and false by another, we will say the predicate has the truth value suspend, indicating that some work has been done on it. We are assumeing all processor are working with all clauses.

After careful consideration, it will become clear that $\perp$ belongs in the scheme. We will be determining truth value assignments based on the operations of several processors and our intuition leads to the naturalness of assigning $\perp$ to an instantiated predicate in the following cases: if a processor has not even begun dealing with its values yet; if more "work" is needed before assigning it a truth value and this work can proceed without interfering or contradicting the operation of the other processors (Belnap, 1977).

More formally, along the lines of Fitting and Ben-Jacob (1990) we get the following definition:

Five is the space of truth values $\{\mathrm{T}, \mathrm{t}, \mathrm{f}$, suspend, $\perp\}$ with the ordering $<5$, where $\perp<5$, suspend $<5, \mathrm{f}<5$, $\mathrm{\top}$ and $\perp<5$ suspend $<5, \mathrm{t}<5 \mathrm{~T}$. Figure 1 illustrates the ordering pictorially.

Clearly, the ordering $<5$ is based on the amount of information or knowledge available. We note the existence of an alternative ordering $<5 *$ where $\mathrm{f}<5 *$ suspend $<5 * \mathrm{t}, \mathrm{f}<5 * \mathrm{~T}<$ $5 * \mathrm{t}$ and $\mathrm{f}<5 * \perp<5 * \mathrm{t}$. The ordering $<5 *$ is based on the amount of truth available.

The extension of interpretations from atomic formulae, e.g. $\mathrm{A}$ and $\mathrm{B}$, to all closed formulae is governed by the truth tables, Tables 1 and 2.

Other truth tables are logically equivalent to combinations of

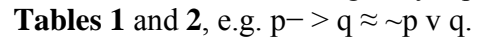

\section{Conclusion}

Today's world of providing us so much information so quickly compels us to rethink what is meant by the options representing possible states of computation (Milner, 2009). Human and computer problem solving is often done in parallel.

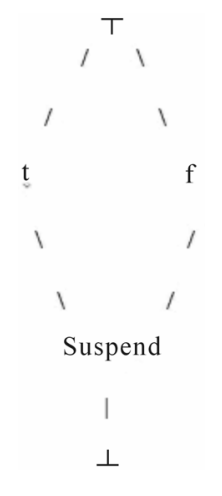

Figure 1.

FIVE: the pace of true values.

Table 1.

Truth values for $\mathrm{A} v \mathrm{~B}$ and $\mathrm{A} \cap \mathrm{B}$.

\begin{tabular}{cccc}
\hline A & B & A v B & A $\cap$ B \\
\hline true & false & t & $\mathrm{f}$ \\
& true & t & t \\
& suspend & t & suspend \\
false & $\perp$ & t & $\perp$ \\
& false & f & $\mathrm{f}$ \\
& suspend & suspend & $\mathrm{f}$ \\
& $\perp$ & $\perp$ & $\mathrm{f}$ \\
suspend & suspend & suspend & suspend \\
& $\perp$ & $\perp$ & $\perp$ \\
$\perp$ & $\perp$ & $\perp$ & $\perp$ \\
\hline
\end{tabular}

Table 2.

Truth values for $\sim$ A.

\begin{tabular}{cc}
\hline $\mathrm{A}$ & $\sim \mathrm{A}$ \\
\hline true & false \\
false & true \\
suspend & suspend \\
$\perp$ & $\perp$ \\
\hline
\end{tabular}


Our contention is that the aforementioned multi-valued logic addresses the concerns attached to both our present day technology and human thought. In order to insure the academic and future professional success of our students they need to be aware of the power of technology and its implications.

\section{REFERENCES}

Adobe Systems (2011). Adobe systems. http://www.adobe.com/

Belnap Jr., N. D. (1977). A useful four-valued logic. In Dunn and Epstein (Eds.), Modern uses of multiple valued logic (pp. 8-37). Dordrecht: Reidel.

Ben-Jacob, M. (2012). Learning: Lifelong, life-wide, sustainable development and the traditional classroom. In Proceedings of the International Conference on Learning and Community Enrichment (ICOLACE).

\section{Singapore.}

Blackboard (2011). Blackboard Inc. http://www.blackboard.com/ Blogs.com (2011). Six apart. www.blogs.com

Davis, R. (2010). Did You KnowProceedings of the Global Learn Asia Pacific Conference

Elluminate (2011). Elluminate Inc. http://www.elluminate.com/

Fitting, M. C., \& Ben-Jacob, M. (1990). Stratified, weak stratified, and three-valued semantics. Fundamenta Informatica, 13, 19-33.

Iclicker (2011). iclicker. http://www.iclicker.com/dnn/

Levin, D. (2008). Presentation at Mercy College.

Milner, R. (2009). The space and motion of communicating agents. Cambridge: Cambridge University Press

Taskstream (2011). Taskstream. https://www.taskstream.com/pub/

Twitter (2011). Twitter Inc. http://twitter.com/

Wikipedia (2011). Wikimedia Foundation Inc. http://wikipedia.org/wiki/Main_Page 